\title{
QUEEN'S
UNIVERSITY
BELFAST
}

\section{Online Thévenin Equivalent Determination Using Graphical Phasor Manipulation}

Abdelkader, S. M., Eladi, A. A., Saeed, M. A., \& Morrow, D. J. (2018). Online Thévenin Equivalent Determination Using Graphical Phasor Manipulation. International Journal of Electrical Power and Energy Systems, 97, 233239. https://doi.org/10.1016/j.ijepes.2017.11.013

\section{Published in:}

International Journal of Electrical Power and Energy Systems

\section{Document Version:}

Peer reviewed version

\section{Queen's University Belfast - Research Portal:}

Link to publication record in Queen's University Belfast Research Portal

\section{Publisher rights}

Copyright 2017 Elsevier.

This manuscript is distributed under a Creative Commons Attribution-NonCommercial-NoDerivs License

(https://creativecommons.org/licenses/by-nc-nd/4.0/), which permits distribution and reproduction for non-commercial purposes, provided the author and source are cited

\section{General rights}

Copyright for the publications made accessible via the Queen's University Belfast Research Portal is retained by the author(s) and / or other copyright owners and it is a condition of accessing these publications that users recognise and abide by the legal requirements associated with these rights.

Take down policy

The Research Portal is Queen's institutional repository that provides access to Queen's research output. Every effort has been made to ensure that content in the Research Portal does not infringe any person's rights, or applicable UK laws. If you discover content in the Research Portal that you believe breaches copyright or violates any law, please contact openaccess@qub.ac.uk. 


\title{
Online Thévenin Equivalent Determination Using Graphical Phasor Manipulation
}

\author{
Sobhy M. Abdelkader ${ }^{1}$, Abdelfattah A. Eladl ${ }^{1}$, Mohammed A. Saeed ${ }^{1}$, and D John Morrow ${ }^{2}$ \\ ${ }^{1}$ Electrical Engineering Department, Faculty of Engineering, Mansoura University, 35516 Egypt \\ 2 School of Electronics, Electrical Engineering and Computer Science, Queen's University Belfast, Northern Ireland \\ E-mail: eladle7@mans.edu.eg
}

\begin{abstract}
This paper presents a new method for determining the Thévenin equivalent parameters of a power system at a node. A graphical method for deriving Thévenin equivalent parameters using online PMU measurements is developed based on representing and manipulating the measured voltage phasors in the complex voltage plane. The proposed method has the ability to use a large numbers of measurements for individual calculation thus providing greater tolerance of bad and erroneous measurements. The method is both computationally efficient and displays improved performance over similar methods when implemented using actual PMU data.
\end{abstract}

Keywords — Phasor measurement, power system, Thévenin Equivalent.

\section{INTRODUCTION}

What a whole world of fantastic applications could be implemented locally by having an accurate equivalent of the power system at a node?! Dynamic short circuit level monitoring, voltage stability assessment, capability to accommodate variable generation sources, etc. are just some of a long list of operation, monitoring and control functions that can be implemented locally if such an accurate equivalent was available online. This may explain the lasting and increasing interest in determining a Thévenin Equivalent (TE) of a power system at a node and the resulting wide variety of methods for TE determination and the applications for which it may be used [1-14].

TE has been used for electromagnetic transients analysis [1], voltage stability assessment $[2,4,7,11]$, harmonic analysis [3], analysis of faulted system [5], designing an adaptive under voltage load shedding [6], constructing node capability chart [8], estimating maximum power transfer limits [10], and developing an adaptive fault location algorithm [14]. Different approaches have been used for determining TE parameters. Time-domain [1], multiple power flows solutions [2], and local phasor measurements using different methods [7, 9, 11-15] are examples. A comprehensive review of the common methods for TE determination and its application can be found in [12, 15]. Reference [16] presented an analogous comparative study of four different TE identification methods (i.e., least squares method, Tellegen's theorem method, adaptive method, and coupled single-port circuit method) based on the time complexity and requirements for PMU availability.

Recently, a method for determining the ac system equivalent TE for transient stability EMT simulation of combined ac/dc power system is introduced in [17]. In [18], local measurements are used to determine the TE online and hence adapt the overcurrent relay to the varying short circuit level. Localized approach is not only having advantage over wide 
area approach, but also it may be the only available approach in some situations where system wide data are not available such as wind developers, DGs, market environment ...etc.

The authors have previously presented two different methods for online determination of the TE using PMU measurements $[12,15]$. In the first [12], the system side was assumed unchanged during the period of 3 measurements and the main purpose of that method was to overcome the phase angle drift in PMU due to system frequency changes. The second method [15] considered both system side changes and measurement errors through using more measurement points and determining a number of possible values of $Z_{\text {th }}$ and subsequently selecting the one with the highest frequency of occurrence. Despite the success of the authors' previous two methods and similar methods [9], the authors believe that there is still room for improvement particularly when applied to real system measurements that inevitably contain some erroneous data. The larger the number of measurements used the higher the probability of a more accurate estimate of the TE parameters. The method in [12] is limited to 3 measurements and therefore there is the considerable possibility of incorrect estimation of the $\mathrm{Z}_{\text {th }}$ if there should happen to be system side changes during the measurement period. While there is no limit on the number of measurements used in [15], however as the number of measurements used gets larger the frequency distribution of $Z_{\text {th }}$ becomes more diverse with different $Z_{\text {th }}$ values expressing similar probability values. This makes the identification of a reliable estimate of $\mathrm{Z}_{\mathrm{th}}$ much more difficult if not impossible.

It is true that the greater the number of manipulations performed on the measurements the greater the chance for measurement error spreading and hence a detrimental effect on the determined $Z_{\text {th }}$. The method of [12] derives some quantities from the measured phasors, which are then used to synchronize the measurements and determine phase angle corrections. Phasors with corrected values are subsequently used to determine the TE parameters thus making four consecutive manipulation steps. The method of [15], uses almost the same number of steps to determine the coefficients of $\mathrm{R} / \mathrm{X}$ linear relationship coefficients, determine the line intersection points, construct the frequency distributions of $\mathrm{Z}_{\mathrm{th}}$, and determining $\mathrm{Z}_{\mathrm{th}}$. This again gives rise to the possibility of measurement error spreading and accumulation.

It may become clear from the above discussion that a method for determining the TE parameters of a power system that can use a large number of measurements and which involves fewer calculations is highly desirable. This was the motive for the authors to develop the method presented in this paper where measured voltage phasors are presented in the complex voltage plane and a circle is constructed through the end points of these phasors, from which the TE parameters are determined. Unlike the techniques reported in [12] and [15] no preprocessing of the measured voltage phasor is required and the only assumption is that the power factor at the measurement point is constant or subject to only small variations during the measurement period. The validity of this assumption has been indicated through investigating of a large number of real measurements at different locations in Northern Ireland. Figs 1.a and 1.b depicts the power factor measured at two different locations, which clearly show the very small variations in power factor 
during the measurement period of 5 minutes or 15,000 measurements. Fig 1.c shows the power factor measured at a point following a disturbance. It may be observed that even under such conditions the power factor remains relatively constant.

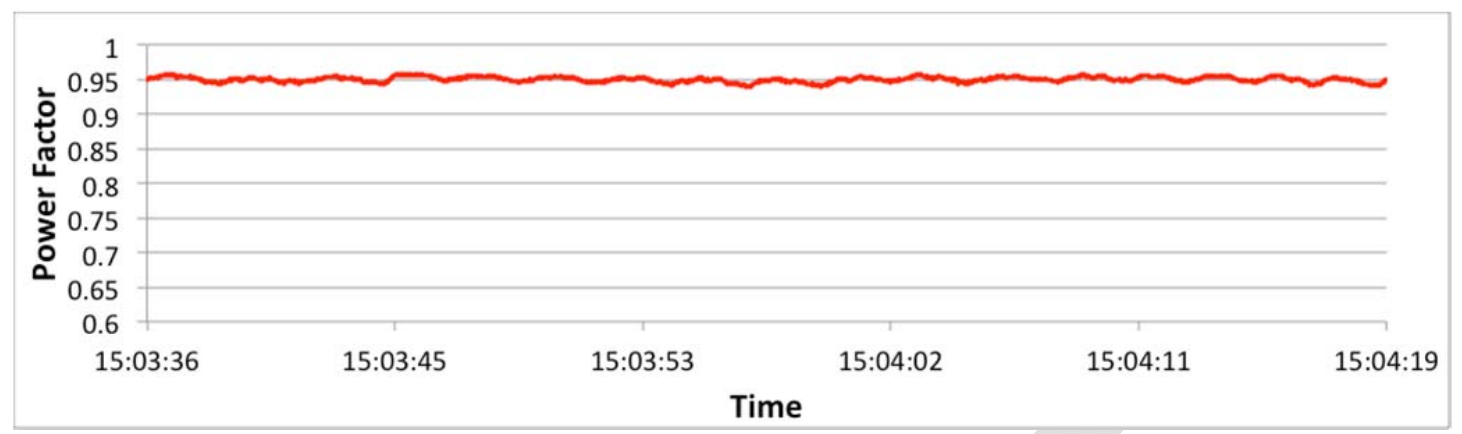

\section{a. Power factor during steady state conditions}

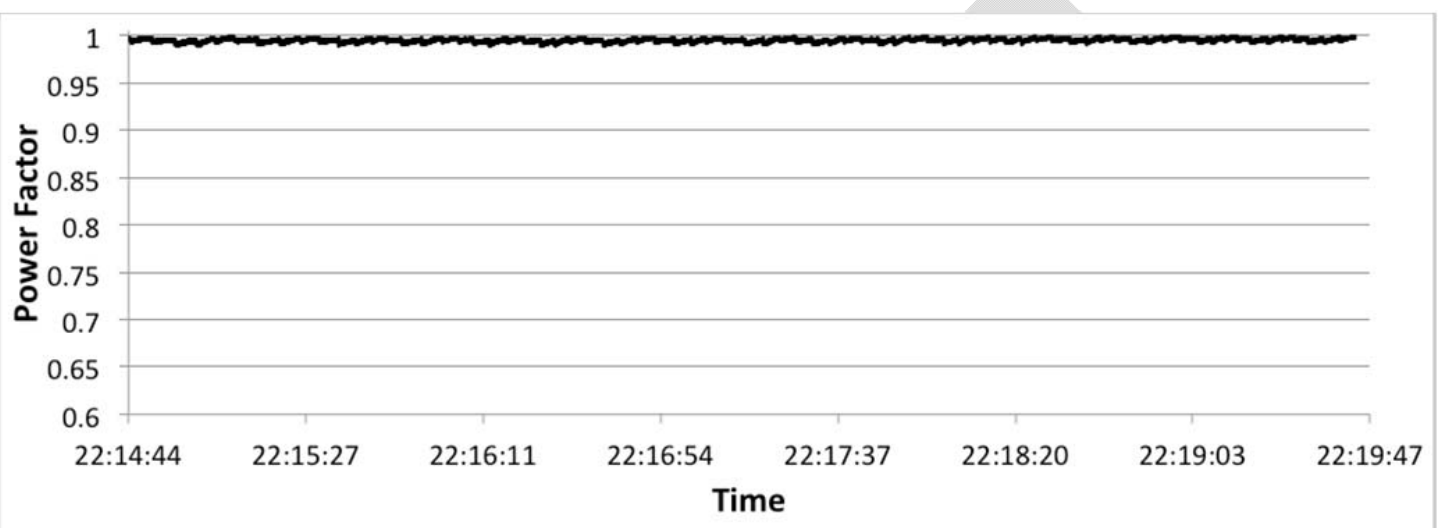

\section{b. Power factor during steady state conditions}

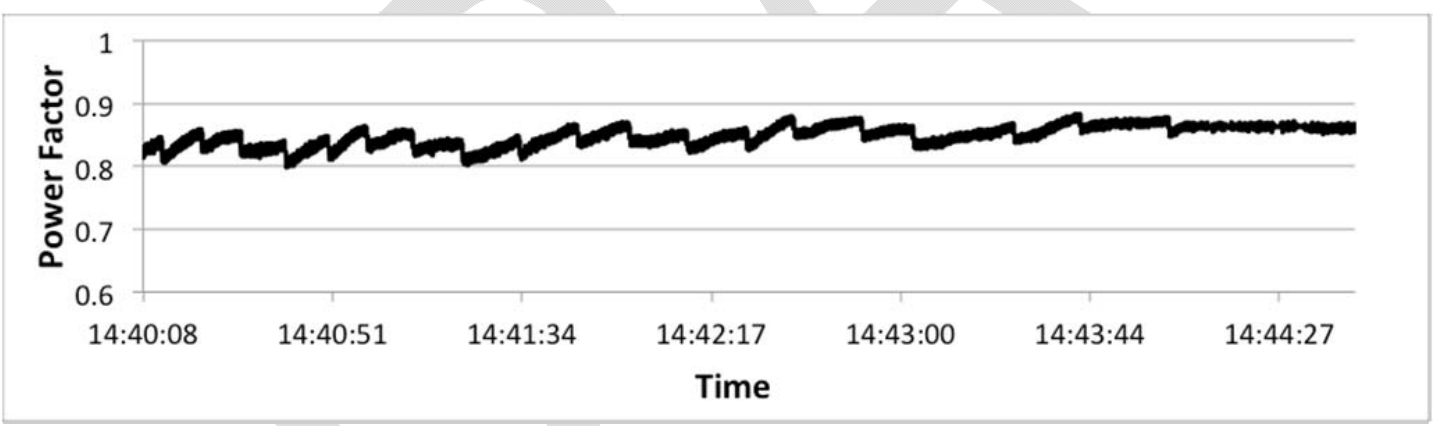

c. power factor measured during a disturbance

Fig. 1. Power factor measured at a power system node

\section{The Proposed Method}

The proposed method is based only on the assumption that the power factor of the power transfer at a node remains constant or varies within very small band during a relatively short time interval, of the order of several minutes. This assumption is justified by real measurements at different locations over long periods as shown above. In such a case, the locus of voltage phasors in the complex voltage plane will be a circle with its parameters such as radius and center coordinates, being representative of the TE parameters. Hence, if a circle is constructed through the measured voltage phasors, the TE parameters for the system can be determined. The advantage of this method is the ability to use a large number of measurements in one TE determination thereby reducing sensitivity to bad and erroneous data.

The following sections present the theoretical foundations of the new method including mathematical proof of the 
voltage phasor locus and derivation of the TE parameters from the geometric parameters of that locus. The calculation procedure is then explained followed by a verification of the proposed method using simulated data of the IEEE 30 bus standard test system.

\subsection{Theoretical Foundations}

For a simple system consisting of a source having an EMF of magnitude $\mathrm{E}$ and an impedance $\mathrm{Z}$ supplying a load $\mathrm{P}+\mathrm{jQ}$, the active and reactive power equations can be expressed as follows.

$$
\begin{aligned}
& P=\frac{V E}{Z} \cos (\delta+\theta)-\frac{V^{2}}{Z} \cos (\theta) \\
& Q=\frac{V E}{Z} \sin (\delta+\theta)-\frac{V^{2}}{Z} \sin (\theta)
\end{aligned}
$$

where $\mathrm{V}$ is the terminal voltage magnitude, $\delta$ is the terminal voltage angle with respect to the direction of $\mathrm{E}$, and $\theta$ is the impedance angle. With a phase angle difference $\varphi$ between current and voltage at the terminal bus, reactive power and reactive power are related as follows.

$$
Q=\tan (\varphi) P
$$

Substituting for P and Q from (1) and (2) into (3)

$$
\frac{V E}{Z} \sin (\delta+\theta)-\frac{V^{2}}{Z} \sin (\theta)=\tan (\varphi)\left(\frac{V E}{Z} \cos (\delta+\theta)-\frac{V^{2}}{Z} \cos (\theta)\right)
$$

With some straightforward manipulations (4) can be put in the following form

$$
\left(V \cos (\delta)-\frac{E}{2}\right)^{2}+\left(V \sin (\delta)-\frac{E}{2 \tan (\theta-\varphi)}\right)^{2}=\left(\frac{E}{2 \sin (\theta-\varphi)}\right)^{2}
$$

This represents a circle in the voltage plane, considering the direction of $E$ the real axis, with its center at $(E / 2, E /(2$ $\tan (\theta-\varphi))$ and a radius equal to $\mathrm{E} /(2 \sin (\theta-\varphi))$ as shown in Fig. 2. Hence, it can be stated that while the power factor at a node in the system is constant, a single circle can be constructed so as to fit to any set of voltage phasors measured at that node, provided that the rest of the system is unchanged. The circle parameters, radius and coordinates of the center are related to the TE parameters of the system at that node, as it is clear from (5). This represents the basis of the proposed method. It is to be noted that fitting a circle to the measured voltage phasors makes the sensitivity of calculated $\mathrm{TE}$ parameters to measurement errors and to system side variations much lower than that of the $\mathrm{TE}$ parameters calculated using the simple linear relationship used in [12] and [15].

It worth mentioning that if the TE parameters for a system are known it is straightforward to construct the circle representing the voltage locus for a specified power factor. The reverse process, i.e. deducing the TE parameters from a circle constructed to the measured voltage phasors is not as straightforward. This is because of the fact that the circle fitted to a group of measured voltage phasors has no information about the direction and magnitude of E, which is the reference for the key points on the standard circle of Fig. 2. Therefore, the determination of the direction E is essential 
for determining the TE parameters from the circle constructed from the measured voltage phasors.

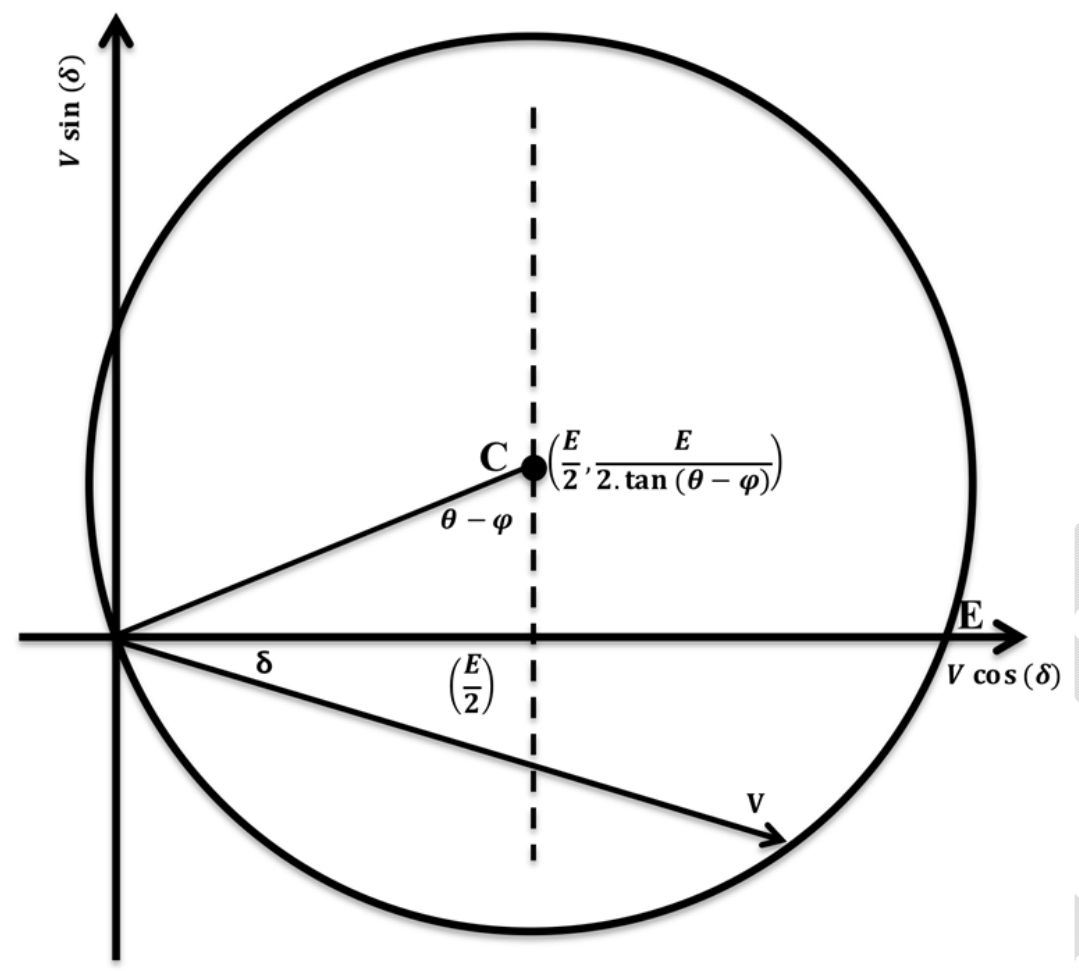

Fig. 2. Constant power factor locus in the complex voltage plane

The direction of $\mathrm{E}$ is obtained by determining the angle, which $\mathrm{E}$ makes with one of the measured voltage phasors, using two pairs of voltage and current phasors obtained from the PMU data. To show how this angle and hence the TE parameters are determined, consider the phasor diagram, Fig. 3, of a power system represented by its TE equivalent at the measurement bus $(\mathrm{E}$ and $\mathrm{Z}=\mathrm{R}+\mathrm{jX})$; the measured voltage, $\mathrm{V}$, is considered reference and the measured current is $\mathrm{I} \angle-\varphi$.

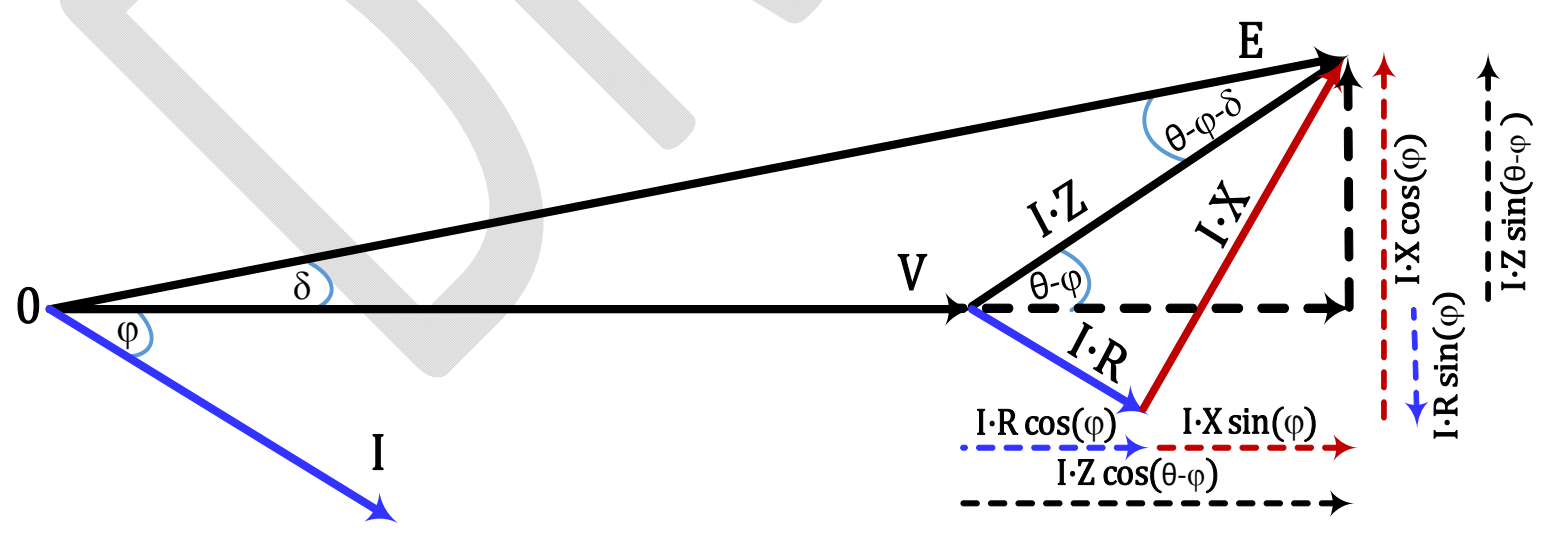

Fig. 3. Phasor diagram of the equivalent system

From the geometry of the phasor diagram and according to the sine rule applied to the triangle formed by the three vectors V, E and I.Z, it can be stated that

$$
\frac{V}{\sin (\theta-\varphi-\delta)}=\frac{E}{\sin (\pi-(\theta-\varphi))}=\frac{I . Z}{\sin (\delta)}=D,
$$


Where V, E and I.Z are the magnitudes of the corresponding vectors and D is the diameter of the circle passing through the ends of the three vectors. It can be noted from (6) that if E, $\theta, \mathrm{Z}$ and $\varphi$ are constants, the diameter D will be the same for all values of $\mathrm{V}$, I and $\delta$. This means that one circle will pass through ends of all voltage vectors. Hence, the angle $\delta$ between $\mathrm{E}$ and any of the voltage phasors will suffice for the purpose of determining the TE parameters. However, in this work, the voltage phasor with least fitting error is used for this calculation. To determine the direction of E, consider again the phasor diagram of Fig. 3. It is evident that

$$
Z . I \sin (\theta-\varphi)=E \sin (\delta)
$$

For two different measurement pairs $\left(\mathrm{V}_{1}, \mathrm{I}_{1}\right),\left(\mathrm{V}_{2}, \mathrm{I}_{2}\right)$ having the same power factor, the same angle $\varphi$ between voltage and the corresponding current, and $V_{1}$ leading $V_{2}$ by angle $\varepsilon_{12}$, the following relationships can be established

$$
\begin{aligned}
& Z . I_{1} \sin (\theta-\varphi)=E \sin (\delta) \\
& Z . I_{2} \sin (\theta-\varphi)=E \sin \left(\delta+\varepsilon_{12}\right)
\end{aligned}
$$

Where $\delta$ is the angle between $\mathrm{E}$ and $\mathrm{V}_{1}$.

Diving (8) by (9), then

$$
\delta=\tan ^{-1}\left(\frac{I_{1} \sin \left(\varepsilon_{12}\right)}{I_{2}-I_{1} \cos \left(\varepsilon_{12}\right)}\right)
$$

If the center of constant power factor circle is located at the point $\left(\mathrm{x}_{\mathrm{c}}, \mathrm{y}_{\mathrm{c}}\right)$ in the voltage plane, its diameter, $\mathrm{D}$, can be determined as

$$
D=2 \sqrt{x_{c}^{2}+y_{c}^{2}}
$$

The magnitude and angle of $\mathrm{Z}_{\mathrm{th}}, \mathrm{Z}$ and $\theta$, can be derived from (6) as in (12) and (13) respectively.

$$
\begin{aligned}
& Z=\frac{D \sin (\delta)}{I_{1}} \\
& \theta=\varphi+\delta+\sin ^{-1}\left(\frac{V_{1}}{D}\right)
\end{aligned}
$$

The magnitude of $E_{\text {th }}$ can then determined as

$$
E=D \sin (\pi-(\theta-\varphi))
$$

\subsection{The TE calculation procedure}

The TE is determined from PMU measurements by the following method.

1. Each voltage phasor is represented by the coordinates of its end point in the complex voltage plane. A circle is constructed through the end points of voltage phasors from a set of consecutive measurements. Any number of points greater than three can be used; however in this work, 50 points are used representing a measurement duration of one second. The center of the constructed circle $\mathrm{C}\left(\mathrm{x}_{\mathrm{c}}, \mathrm{y}_{\mathrm{c}}\right)$ and its diameter are determined. The 
diameter and center of the circle are obtained from the circle fitting algorithm [19] which are used along with equations (11), (5) to determine TE parameters.

2. The phase angle of $\mathrm{E}_{\mathrm{th}}$ is determined using equation (10), bearing in mind that $\angle E_{t h}=\delta+\angle V_{1}$, employing any two measurements. Preference should be given to those measurements which exhibit the least fitting errors to the voltage locus determined by equation (15)

$$
\sqrt{\left(C_{x}-V_{x}\right)^{2}+\left(C_{y}-V_{y}\right)^{2}}=\frac{D}{2}
$$

3. Determine the equivalent impedance magnitude and angle using (12) and (13) respectively.

4. Determine the magnitude of the TE EMF using (14)

\section{3. . Validation with Standard System Data}

To check the validity of the proposed method a data set was extracted from several power flow solutions of the IEEE 30 bus standard test system. Load at bus 30 was varied in $20 \%$ steps from $20 \%$ to $460 \%$ of the standard value of 10.6 $\mathrm{MW}+\mathrm{j}$ 1.9 MVAr. Phase angles of the generators were fixed as per the standard case. Voltage and current phasors at bus 30 for each loading condition are listed in Table 1.

Table 1

\begin{tabular}{llll}
\multicolumn{5}{c}{ Voltage and Current Phasors at Bus 30 oF IEEE 30 Bus System } \\
\hline \hline Mag(V) & Angle(V) & Mag(I) & Angle(I) \\
\hline 1.0395 & -9.76 & 0.0207 & -19.92 \\
1.0315 & -10.46 & 0.0418 & -20.62 \\
1.0231 & -11.17 & 0.0632 & -21.33 \\
1.0145 & -11.89 & 0.0849 & -22.05 \\
1.0056 & -12.63 & 0.1071 & -22.79 \\
0.9962 & -13.39 & 0.1297 & -23.55 \\
0.9865 & -14.16 & 0.1528 & -24.32 \\
0.9764 & -14.95 & 0.1765 & -25.11 \\
0.9657 & -15.77 & 0.2007 & -25.93 \\
0.9546 & -16.61 & 0.2256 & -26.77 \\
0.9429 & -17.47 & 0.2513 & -27.63 \\
0.9305 & -18.37 & 0.2777 & -28.53 \\
0.9175 & -19.3 & 0.3052 & -29.46 \\
0.9035 & -20.27 & 0.3337 & -30.43 \\
0.8887 & -21.3 & 0.3635 & -31.46 \\
0.8726 & -22.38 & 0.3949 & -32.54 \\
0.8552 & -23.53 & 0.4281 & -33.69 \\
0.8361 & -24.77 & 0.4637 & -34.93 \\
0.8147 & -26.13 & 0.5023 & -36.29 \\
0.7903 & -27.65 & 0.5450 & -37.81 \\
0.7613 & -29.41 & 0.5941 & -39.57 \\
0.7242 & -31.61 & 0.6542 & -41.77 \\
0.6651 & -35 & 0.7448 & -45.16 \\
0.6327 & -36.81 & 0.7919 & -46.97 \\
\hline \hline & & & \\
\hline
\end{tabular}

Fig. 4 depicts the complex voltage plane with the recorded voltage phasors presented by the circular marker. The figure also shows the circle constructed through the voltage phasors with its center $\mathrm{C}$ at $(0.5667,0.2305)$ and its radius $\mathrm{R}=0.612 \mathrm{pu}$. The figure shows that almost all the voltage phasors ends are lying on the constructed circle. Phasors $\mathrm{V}_{1}$ 
and $\mathrm{V}_{2}$ on the figure have however the best fit and hence are used to determine the TE parameters. These are found to be: magnitude of $E_{t h}, E=1.0475 \mathrm{pu}$, and its angle is $-9.02^{\circ}$. Magnitude of $Z_{\text {th }}$ is found to be 0.7179 and its angle is $69.01^{\circ}$. These values are almost the same as those determined in $[2,12,15]$ for the same system under the same conditions. It worth mentioning that the calculated TE voltage, E, is found to be exactly the same as that determined from load flow calculations with the load at bus 30 set to zero and the voltage angles of the generators fixed at their standard case values.

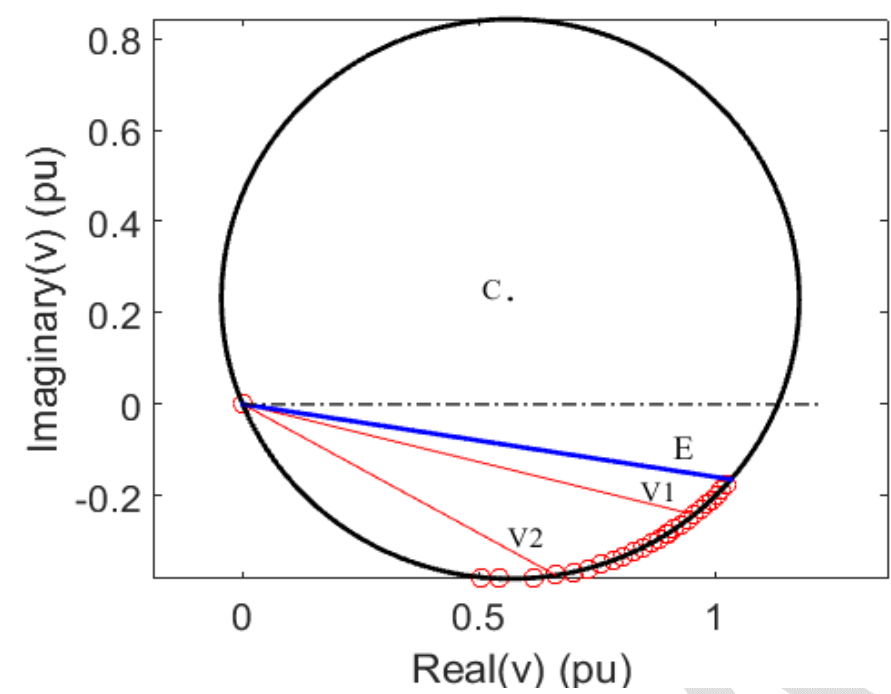

Fig. 4. Voltage phasors, the fitted circle and $\mathrm{E}_{\text {th }}$ for bus 30 of the IEEE 30 bus system

To test the effect of measurement inaccuracy on the estimated TE, errors were intentionally introduced to the data set of Table 1; four different cases with errors ranging from $2 \%$ to $5 \%$ were tested. The theoretical measurement errors introduced to the data set is assumed to model the probability of the occurrence of practical measurements errors. Fig. 5 shows the graphs obtained for the four cases superimposed on the original case. Slight differences may be observed between the circles constructed for each case. These very slight differences do not however have an appreciable effect on the estimated TE parameters and the E vectors of the 4 test cases are almost the same as for the standard case.

For the sake of comparison, the number of erroneous impedance values for the method of [15] is calculated for different numbers of the measurements used in each calculation step and different numbers of bad measurements. Fig. 6 shows the percentage erroneous values of the total number of the estimated $\mathrm{Z}_{\text {th }}$ values. In this figure, the lower curve is for one bad measurement; it can be observed that the erroneous $Z_{\text {th }}$ values is 100 percent of the total $Z_{\text {th }}$ values calculated using 4 measurements including the one bad measurement. This percentage gets lower as the number of measurements used increases; $50 \%$ for 10 measurements and $25 \%$ for 25 measurements.

The presence of two bad measurements, the middle curve in Fig. 6 implies that the number of erroneous calculated $\mathrm{Z}_{\mathrm{th}}$ becomes $40 \%$ of the total values determined using 25 measurements. No correct $\mathrm{Z}_{\mathrm{th}}$ can be determined using the five measurements used in [15]. Even with 25 measurements, the erroneous $Z_{\text {th }}$ values are approximately $55 \%$ of the total as can be observed from the upper curve of Fig. 6 . This of course will reflect in the final value of $Z_{\text {th }}$ determined by 
averaging all the estimated $Z_{\text {th }}$ values. Unless the bad measurements are detected and removed, the estimated $Z_{\text {th }}$ would have a considerable error that compromises its value.

It may become clear that the proposed method has more capability to tolerate bad measurements than the previous methods introduced by the authors $[12,15]$. In the following section, the results of application of the proposed method to real measurements are compared with those obtained using the method of [15].

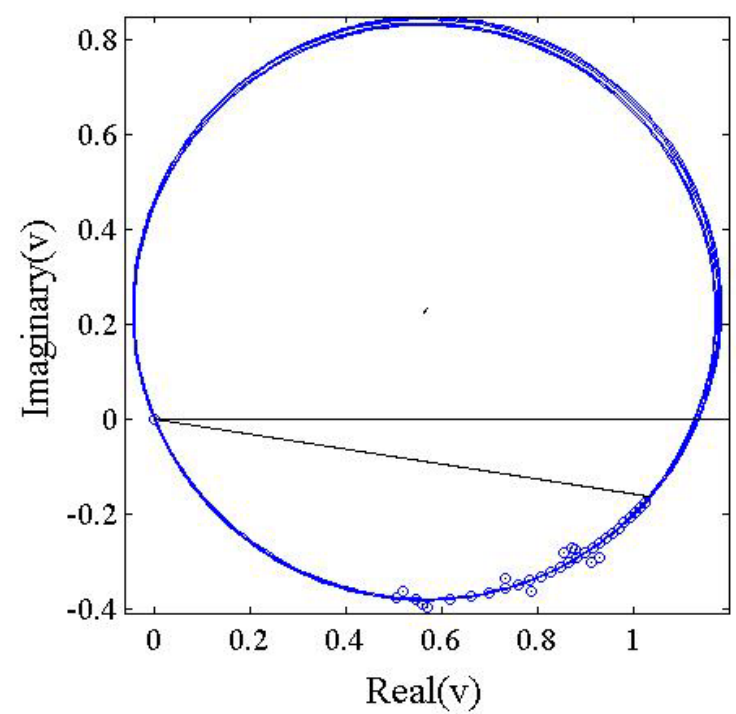

Fig. 5. Voltage phasors, the fitted circle and $\mathrm{E}_{\mathrm{th}}$ for bus 30 of the IEEE 30 bus system

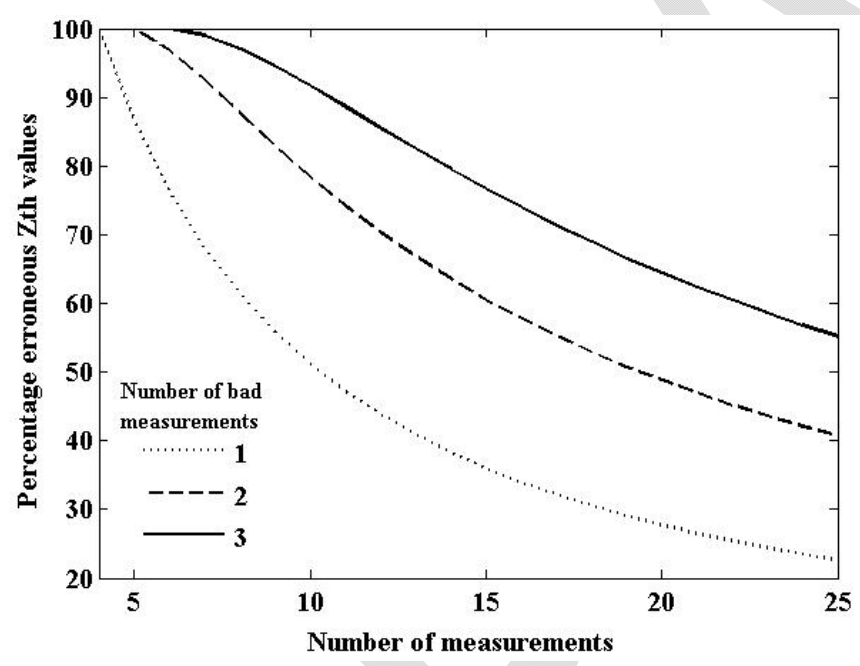

Fig.6 Percentage erroneous impedance values

\section{Application Results AND DisCussion}

The proposed method has been applied to PMU measurements recorded at the terminals of a wind farm in Northern Ireland. The same set of measurements has been used in [15] and the resulting values of $Z_{\text {th }}$ calculated have been found to be in agreement with the real system parameters reported in [20] and also validated through comparison with the events recorded at the time of measurements. This data set is used in this work for the sake of comparison and clarifying the improvements provided by the proposed method over the previous methods.

The data set includes two consecutive records, each 5 minutes long, which were selected from measurements during arduous weather conditions. The first record has no major incidents; only an unsuccessful reclosing trial took place. 
Therefore, this record would be considered a steady state condition. The second five minute record includes the tripping of the second circuit of a parallel transmission line and the commencement of a generating station run back scheme, which greatly affected the system equivalent parameters; hence this period is considered a transient condition.

The following two subsections present comparisons of the proposed method with the method [15] for steady state operation and then for transient condition.

\subsection{Steady State Operation}

TE equivalent parameters are determined using [15] as well as the proposed method for the steady state period between 23:15 to 23:20. The TE impedance parameters, $r$ and $x$, determined by [15] are shown in Fig. 7.a, whereas those determined by the proposed method are shown in Fig. 7.b. It can be observed that the TE parameters determined by both methods are similar, but those determined by [15] exhibit more variation. The proposed method has produced smoothed traces of the TE parameters variations. This is expected as the proposed method uses a larger number of measurements and the circle thus constructed is more tolerant to bad data than the straight line approach adopted in [15].

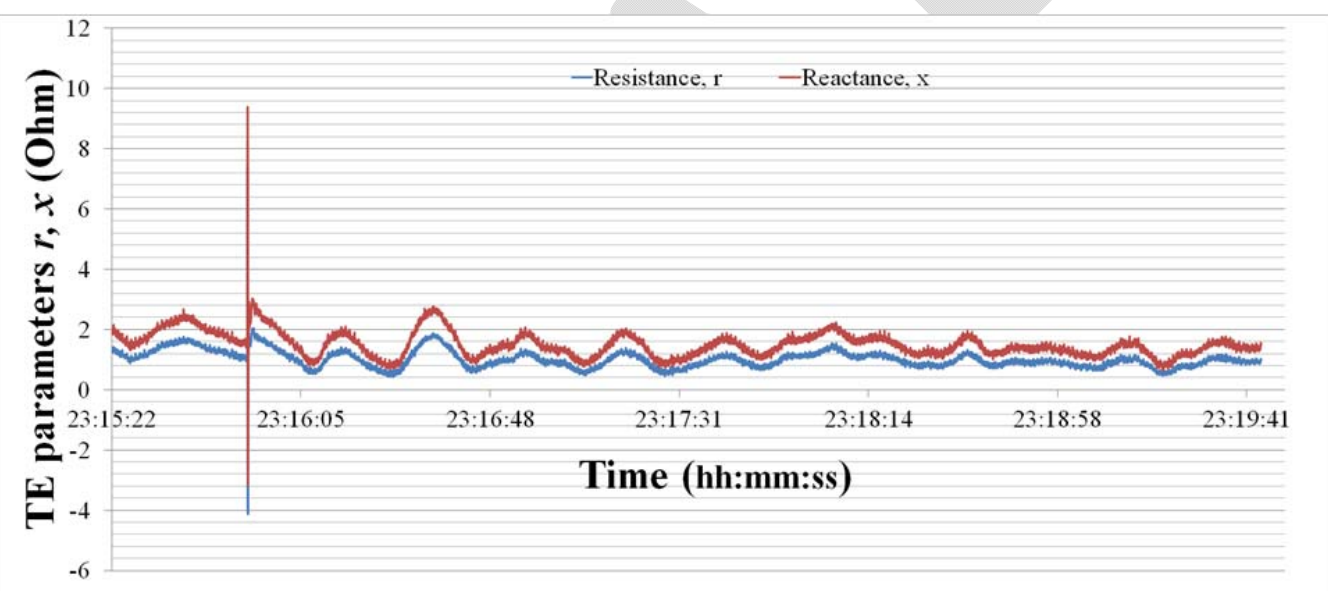

a. Method of [15]

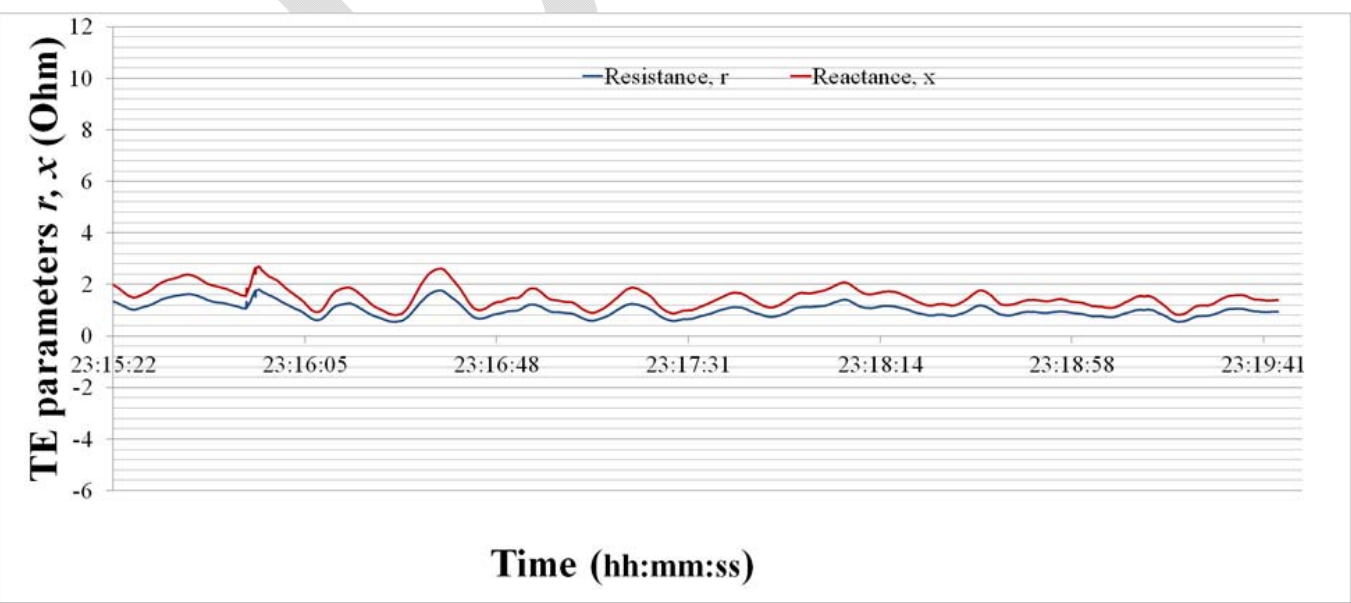

b. Proposed Method

Fig. 7. TE parameters, $r$ and $x$, by two methods for steady state 


\subsection{Transient Condition}

As stated above during the five-minute period between 23:19 to 23:24, the remaining second circuit of a parallel transmission line was tripped out and a run back scheme of a generating station had commenced. The TE parameters during this period have been determined using both [15] and the new method. Figs. 8.a \& 8.b show the estimated TE impedance parameters by [15] and the new method respectively; while Fig 8.c depicts the $\mathrm{E}_{\mathrm{th}}$ estimated by the two methods. Similar to the steady state case, the TE parameters estimated by the new method are smoothed and free of the very short-term variations.

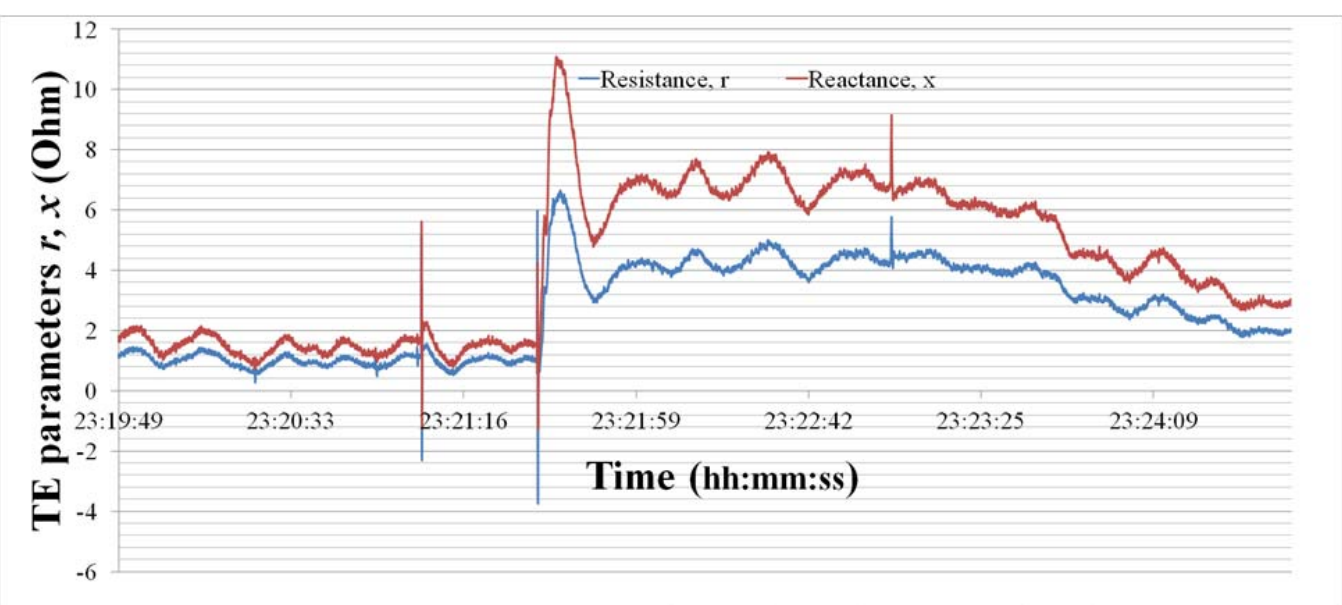

a. The method of [15].
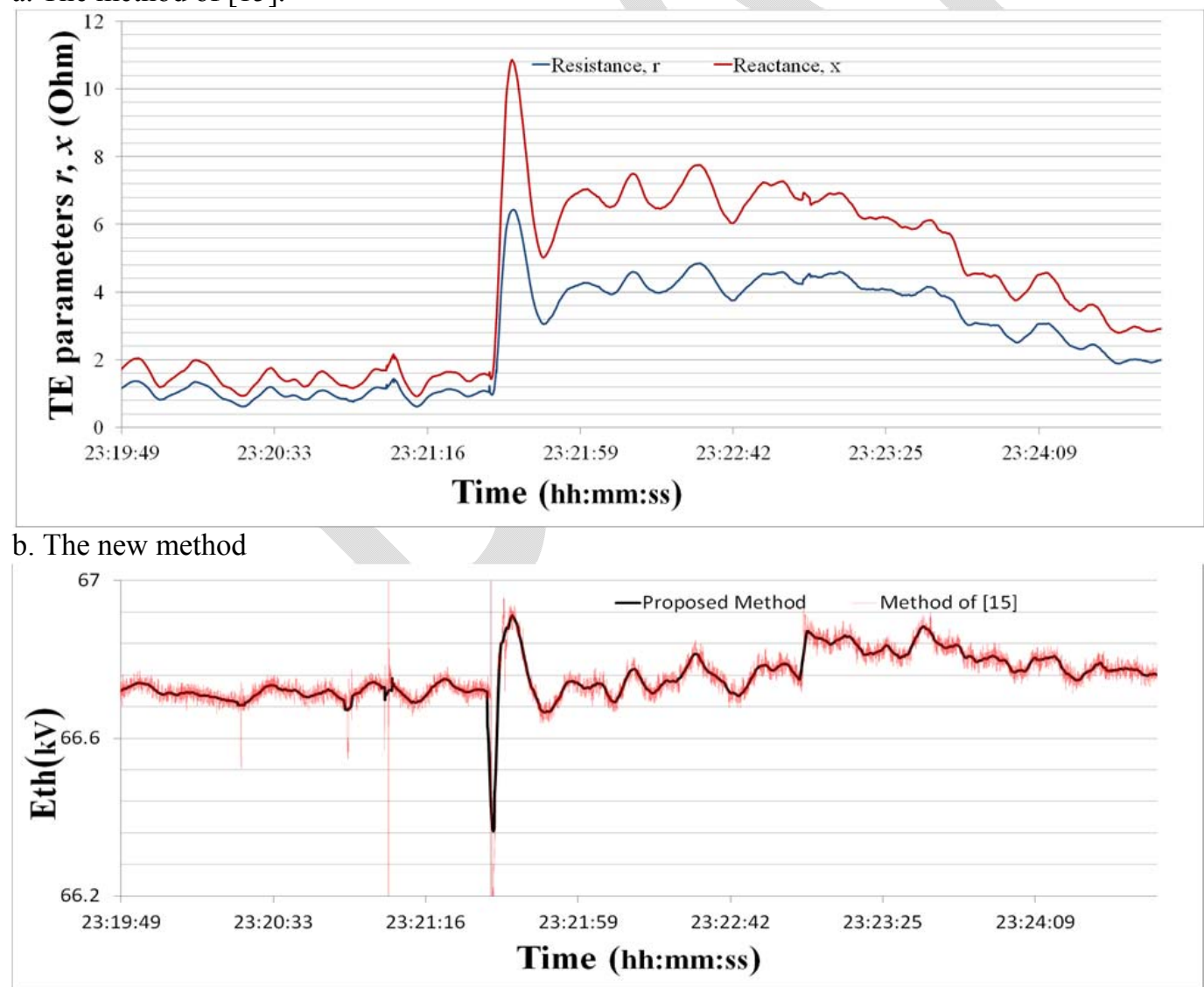

c. $\mathrm{E}_{\mathrm{th}}$ estimated by [15] and the new method

Fig.8. TE parameters, $r \& x$, by two methods for transient condition

Very short transient variations are filtered out as can be observed from Figs 9.a \& 9.b, which show a period of the 
estimated TE parameters $r$ and $x$ respectively by the two methods on a faster time base. It is noteworthy that the spikes due to unsuccessful reclosing disappeared from the parameters estimated by the new method. Also, the short-term oscillations in the parameter values estimated by [15] are filtered out by the new method. However, the long transient variations in both $r$ and $x$ are retained by the new method.
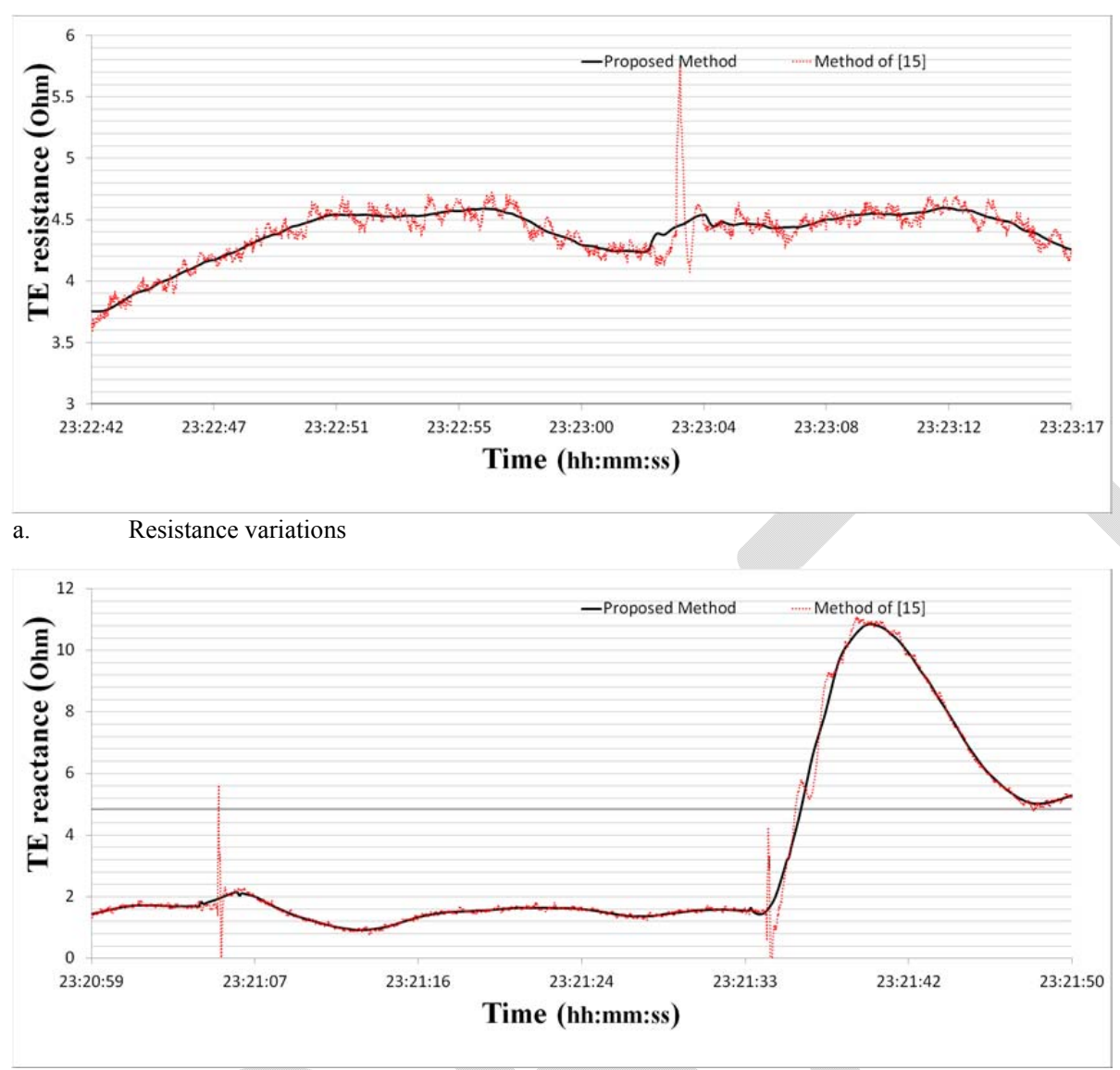

b. Reactance variations

Fig.9. TE parameter zoomed transient variations by the two methods

It worth mentioning that length of the calculation window is the major factor determining what is filtered out and what is retained. In this work, the calculation window length is 50 consecutive measurement points i.e. one cycle. The window size of $1 \mathrm{sec}$ has been arbitrarily chosen by the authors. However, making this choice was based on the objective of filtering out the very fast transients keeping at the same time the slow dynamics while the effects of longer period transient variations are retained. Moreover, this can be tuned according to network requirements and/or further error analysis which is being carried out.

\section{Conclusion}

A method for online determination of the TE parameters of a power system at a node is presented. The method is based on graphical manipulation of the measured voltage phasors where TE parameters are derived from complex voltage plane representation of the measured phasors. The proposed method has the advantages of handling large number of measurements during each calculation, a high degree of tolerance to bad measurements, and the ability to filter short term variations and very short transients that are of less/no interest in the context of TE determination. 
The proposed method has been validated with data from both standard system simulations and real system data from

PMU measurements at the terminals of a wind farm in Northern Ireland. Results of applications have proven the improvement in the TE parameter determination over previous methods.

\section{REFERENCES}

[1] J-H. Hong, and J-K Park, "A Time-domain approach to transmission network equivalents via prony analysis for electromagnetic transients analysis," IEEE Trans. On Power Systems, vol. 10, No. 4, pp.1789-1797, Nov. 1995.

[2] M. M. Elkateb, S. Abdelkader and M. S. Kandil, "Linear Indicator for Voltage Collapse in Power Systems" IEE Proc.-Pt. C., Vol. 144, No. 2, pp.139-146, March1997

[3] S. Abdelkader, M. H. Abdel-Rahman, and M. G. Osman, "A Norton equivalent model for nonlinear loads", In Proc. Large Eng. Syst. Conf. Power Engineering, Halifax, NS, Canada, pp.63 -67, 2001.

[4] I. Šmon, G. Verbi?, and F. Gubina, "Local voltage-stability index using Tallegen's theorm," IEEE Transactions on Power Systems, Vol. 21, No. 3, pp. 1267-1275, Aug 2006.

[5] R. K. Gajbhiye, B. Gopi, P. Kulkarni, and S. Soman, "Computationally efficient methodology for analysis of faulted power systems with series-compensated transmission lines: A phase coordinate approach," IEEE Transactions on Power Delivery, Vol. 23, No. 2, pp. 873-880, Apr 2008.

[6] S.-J. S. Tsai, and K-H. Wong, "Adaptive undervoltage load shedding relay design using Thevenin equivalent estimation", Power and Energy Society General Meeting 2008, DOI: 10.1109/PES.2008.4596370

[7] S. Corsi, and G. N. Taranto, "A real-time voltage instability identification algorithm based on local phasor measurements," IEEE Transactions on Power Systems, Vol. 23, No. 3, pp. 1271-1279, Aug 2008

[8] S. Abdelkader and D. Flynn, "Graphical determination of network limits for wind power integration," IET Gener. Transm. Distrib., 2009, Vol. 3, Iss. 9, pp. 841-849.

[9] S. A Arefifar, and W. Xu, "Online tracking of power system impedance parameters and field experiences," IEEE Transactions on Power Systems, Vol. 24, No. 4, pp. 1781-1788, Oct 2009.

[10] W. Li, Y. Wang, and T. Chen, "Investigation on the Thevenin equivalent parameters for online estimation of maximum power transfer limits," IET Gener. Transm. Distrib., Vol. 4, No. 10, pp. 1180-1187, 2010.

[11] W. Xu, I. R. Pordanjani, Y. Wang, and E. Vaahedi, “A network decoupling transform for phasor data based voltage stability analysis and monitoring," IEEE Trans. On Smart Grid, vol. 3, No. 1, pp.261-270, March 2012.

[12] S. M. Abdelkader and D. J. Morrow, "Online tracking of Thévenin equivalent parameters using PMU measurements,” IEEE Trans. Power Syst., vol. 27, no. 2, pp. 975-983, May 2012.

[13] A. H. Almohammed and M. A. Abido, "An adaptive fault location algorithm for power system networks based on synchrophasor measurements,” Electric Power Systems Research, Vol. 108, pp. 153-163, March 2014.

[14] A. H. Almohammed and M. A. Abido, "A Fully Adaptive PMU-Based Fault Location Algorithm for SeriesCompensated Lines,” IEEE Trans. Power Syst., vol. 29, no. 5, pp. 2129-2137, Sep. 2014.

[15] S. M. Abdelkader and D. J. Morrow, "Online Thevenin equivalent determination considering system side changes and measurement errors,” IEEE Trans. Power Syst., vol. 30, no. 5, pp. 2716-2725, Sep 2015.

[16]H. Yuan and F. Li, "A comparative study of measurement-based Thevenin equivalents identification methods," 2014 North American Power Symposium (NAPS), Pullman, WA, 2014, pp. 1-6.

[17] A. A. van der Meer, M. Gibescu, M. A. M. M. van der Meijden, W. L. Kling, and J. A. Ferreira, "Advanced hybrid transient stability and EMT simulation for VSC-HVDC systems," IEEE Trans. Power Delivery, vol. 30, no. 3, pp. 1057-1065, June. 2015.

[18]A. Rahmati, M. A. Dimassi, R. Adhami, and D. Bumblaukas, “An overcurrent protection relay based on local measurements,” IEEE Trans. On Industry Applications, vol. 51, no. 3, pp. 2081-2085, June. 2015.

[19] http://uk.mathworks.com/matlabcentral/fileexchange/5557-circle-fit

[20] SONI and EirGrid, "Ten year transmission forecast statement," 2013[Online].Available: http://www.soni.ltd.uk/media/documents/Opera-tions/All-Island/Tenyeartransmssionforecaststatement2013.pdf. 